\title{
On the Value of Bones as Diagnostic Material of Fetal Age.
}

\author{
By \\ Dr. Giichiro Takata. \\ (From the Institute for Forensic Medicine of the Chiba \\ Medical School, Chiba, Japan)
}

Many a natural scientist studied the development of the human bones, which seems, however, in recent years to be more or less neglected. Practically we have often to diagnose the age of the fetus from a skeleton or even some pieces of bones, but we cannot find any literature thereof, Excepting that cised from Kanzler's "gerichtlich medizinische SkeletoNekropsie (Casper's Vierteljahresschr. f. gerichtl. Med., 1854) and a few - other original records. As in other studies, the reliable statistic standard of measurements must be established from as many materials as possible. I have therefore intended to summarize those original records, adding my own measurements made on 23 cases.

I selected those bones, whose measurement is simple and of easy access : i.e., ciavicula, scapula, humerus, ulna, radius, femur, tibia, fibula, zygomaticus, maxilla, parietale, squama ossis occipitalis, squama ossis tempolaris, squama ossis frontalis, corpus ossis sphenoidalis and pars basilaris ossis occipitalis (there are many bones of adults which are of easy, but in fetus, of difficult access).

Three of them, i.e., corpus ossis sphenoidalis, pars basilaris ossis occipitalis and os zygomaticum, which were hitherto seldom measured, I, picked out particularly on account of their easy measurableness, while mandibula, costae, vertebrae, ossa pelvis, and other cranial bones than those above cited, (for example the os nasale) ossa metacarpalia, phalanges digitorum manus et pedis and ossa metatarsalia, which were all measured by many authors, I neglected because of their difficulty of orientation or measuring.

Before I-tabulate my results, I will cite the measurements which I found in literature :- 
N.B. 1) For the sake of convenience I used the following abbreviations for the names of authors :

B.=Béclard. G.=Günz. L.=Landois. M.=Meckel. N.=Nicolai. R.=Renault and Rambaud. S.=Senff. Sk.=Skrzeczka. T.=Toldt. U.=Uffelmann. Fr. $=$ French authors, whose names were not mentioned by reporters.

N.B. 2) Nicolai, Senff and Günz used inch and line as units of measurement. There are, however, 3 kinds of inches : the German inch is equal to $27 \mathrm{~mm}$, the French inch to $26 \mathrm{~mm}$. and the English inch $25.5 \mathrm{~mm}$., while the line is recognized as one tenth or one twelfth of an inch. Fearing that conversion may cause errors, I cited the original measurements as such.

N.B. 3) With regard to the length of tubular bones, we know the distinct difference, according to the method of measurement whether including or excluding the cartilageous parts. For the former I marked with stars.

N.B. 4) As to the description of age some used the week or solar month as unit but $I$ have changed all into lunar months. It the designatious such as beginning, middle or end, or earlier or later half, etc. have been dropped off in order to avoid confusion.

N.B. 5) Numbers with (L) in Table I, are different from those fond in the description of Leonard Landois (Ueber das Wachstum der Diaphysen der Röhrenknochen des Menschen während des intrauterinen Lebens.-Virchow's Archiv, Bd. 45 (1869)), and they are those in lunar months obtained by calcuration from Table III.

My materials consist of 23 Japanese of the fetus, i.e., one of the fourth, one of the sixth, 4 of the seventh, 4 of the eighth, 4 of the tenth month and 9 new-born children. For measurement I adopted the Toldt's method (Maschka's Handbuch der gerichtlichen Medizin, 1882, Bd. III, S. 531-534): viz. 
A. Flat bones are measured along the outside by means of a tape measure :

1. The height of the squama ossis occipitalis is measured by the distance between the hind rim of the foramen occipitale magnum and the farthest end of the squama occipitalis. The breadth is no other than the distance between two ends of the sutura mendosa.

2. The distance between the margo frontalis and the occipitalis as measured on a parasagittal line passing through the tuberuclum parietale. The height is measured along the frontal line passing through the tuberclum, indicates the breadth of the parietal bone.

3. The height of the squama frontalis is the distance between the incisura supraorbitalis and the upper end of the bone measured on a line passing through the tuberculum frontale, while its breadth is indicated by the distance between the median line and the sutura coronalis as measured through the tuberculum.

4. The height of the squama tempolaris is measured by the distance between the hind root of the processus zygomaticus and the upper end of the squama, and its breadth by the distance from the incisura parietalis horizontally to the fore end of the squama.

B. Not-flat bones are measured not by means of a tape measure, but in linear distance with a Virchow's measure :

1. The height of the maxilla is measured by the distance from the upper end of its processus frontalis to the inferior end of the septum between the canine and the outer inscisor, and the breadth by the doubled distance from the hind and lower end of its processus zygomaticus perpendicular to the processus palatinus ; for in the fetal bones it is hardly possible to measure the distance between the two zygomatic processes on account of the incompleteness of ossification. 
2. In the case of tubular bones, the distance of both ends the osseous part alone is measured leaving out the cartilageous parts.

3. Though it takes the shape of an $\mathrm{s}$, the clavicula is measured as in other tubular bones.

N.B. Many a author has, measured the beight and breadth of the os parietale. As I have intended to get the average value of as many materials as possible, I have used also the above method. But in this case we can often hardly distinguish the directions of the suturae coronalis et sagittalis causing considerable errors. Hence I prefer rather the Günz's method (cited in CasperLiman, Handbuch der gerichtlichen Medizin, Aufl. 8, Bd. II, S. 912 ; the original report: Günz, Der Leichnam des Nugeborenen, 1827, S. 82), according to which the length of both diagonal lines is measured by means of a tape measure.

C. For those bones whose measuring method was not explained in literature or which were neglected but are important for our purpose, I' selected the following fixed points :

1. For the height of the scapula the line connecting the median angle and the inferior angle is measured along the inside by means of a tape measure, the measuring along the outside is inconvenient by the presence of the spine, and for the breadth along the line connecting the cavitas glenoidalis and the median end of the spina scapulae.

2. For the measurement of the rhombic cheek bone the two diagonal distances of the four angles are measured by means of a Virchow's measure.

3. The length of pars basilaris ossis occipitalis is measured-by the line connecting the fore rim of the foramen occipitale magnum and the free end of the bone, and the breadth by the distance between the two yet unnamed processes which are found in the middle of the two lateral edges.

4. For the length of the corpus of the os sphenoidale the frontooccipitale diameter is measured, and for the breadth the distance between the two tongue shaped unnamed processes on both sides. 
N.B. The letter $m$ in tables IV and $V$ means the fusion of the body with the small wings of the sphenoid bone.

Tables VI and VII indicate the total average of other authors' results as well as my own. For the sake of convenience the inch was taken as $27 \mathrm{~mm}$. and the line as one tenth of an inch.

N.B. The letter c, which is used in the Table VI, denotes the measurements with the cartilageous parts and $o$, those of the osseous part only.

\section{Summary.}

1. The dimensions of one of the two symmetrical bones of one and the same fetus are almost always the same as those of the other (particularly in table IV).

2. Of the fetal bones some grow faster than the others, as will be seen from Table V, VI and VII. The tubular bones as a rule grow faster than the flat skull bones ( $c f$. Table V and VI with Table VI and VII). This may be partly to the inaccuracy of calculating the age and partly to the disproportion between the growth of area or size and that of the weight. The latter seems to be more important as is borne out by the fact that the seeming retardation of growth is met with in flat bones, which undergo marked external changes whereas in the tubular bones the shape does not change much as they grow in weight.

3. In those bones which are not flat or whose external shape does not change much, for example, the pars basilaris ossis occipitalis, the corpus ossis sphenoidalis and the os zygomaticus, the growth is so little that we can not use it for the determination of the fetal age, (tables IV, V and VII).

4.- For the above reason (2) the bones of the extremities are superior to the skull bones as material for deterinining of the fetal age.

I have never heard that the weight of the bones was taken into consideration in order to diagnose the fetal age, but $I$ believe that it is more proper to weigh the bones than to measure them for our purpose. To weigh the bònes we need not to have any specified method; we can easily obtain accurate results even from those bones which are hard to measure for example, the mandibula and the other skull bones of the fetus. 
I have therefore weighed all my material and the results were table VIII, Table IX shows the average weight from the numbers given in the previous table. Weighing was impossible in those cases in which fusion has taken place between the corpus and the alas of the sphenoid bone, and betwen the pars petrosa and squama of the temporal bone, and in which the preparations were intended for denuonstration and are not to be separited into components (these materials were all quite dry).

\section{Conclusion.}

From tables IV, V, VIII and IX, I have arrived at the following conclusions :

1. Increment in dimensions of the fetal bones are not always parallel to that of their weights.

2. One of the symmetrical fetal bones has almost always the same dimensions and weight as the other, with the exception of the inborn defect of the ossification of the parietal bone etc.

3. For the determination of the fetal age from the bones, it is more corract to weigh them than to measure them, becauss the difference in weight is much larger than that of dimension and moreover weighing is as a rule far easier than measuring. 
TABLE I. Bones of the extremities.

\begin{tabular}{|c|c|c|c|c|c|c|c|c|c|}
\hline \multirow{2}{*}{$\begin{array}{l}\text { Fetal } \\
\text { Month }\end{array}$} & \multirow{2}{*}{ Clavicle } & \multicolumn{2}{|c|}{ Scapula } & \multirow{2}{*}{ Humerus } & \multirow{2}{*}{ Radius } & \multirow{2}{*}{ Ulna } & \multirow{2}{*}{ Femur } & \multirow{2}{*}{ Tibia } & \multirow{2}{*}{ Fibula } \\
\hline & & Height & Width & & & & & & \\
\hline II & $\begin{array}{l}1-1.5^{\prime \prime \prime}(\mathrm{N}) \\
1^{\prime \prime \prime}(\mathrm{S}) \\
1-5^{\prime \prime \prime}(\mathrm{B})^{*} \\
2^{\prime \prime \prime}(\mathrm{M})^{*} \\
10 \mathrm{~mm}(\mathrm{R})^{*}\end{array}$ & - & - & $\begin{array}{l}1^{\prime \prime \prime}(\mathrm{N}) \\
1^{\prime \prime \prime}(\mathrm{M}) \\
3^{\prime \prime \prime}(\mathrm{B})^{*} \\
7.4 \mathrm{~mm}(\mathrm{M})^{*}\end{array}$ & $\begin{array}{l}2.2^{\prime \prime \prime}(\mathbf{B})^{*} \\
\operatorname{5mm}(\mathrm{R})^{*}\end{array}$ & $\begin{array}{l}2.5^{\prime \prime \prime}(\mathrm{B})^{*} \\
\operatorname{5mm}(\mathrm{R})^{*}\end{array}$ & $\begin{array}{l}3^{\prime \prime \prime}(\mathrm{B}) \\
8-13 \mathrm{~mm}\left(\mathrm{R},{ }^{*},\right. \\
16 \mathrm{~mm}(\mathrm{~L})^{*}\end{array}$ & $\begin{array}{l}2^{\prime \prime \prime}(\mathrm{B})^{*} \\
25-30 \mathrm{~mm}(\mathrm{R})\end{array}$ & )$^{*} 2^{\prime \prime \prime}(\mathbf{B})^{*}$ \\
\hline III & $\begin{array}{l}3^{\prime \prime \prime}(\mathrm{N}) \\
2^{\prime \prime \prime}(\mathrm{S}) \\
6^{\prime \prime \prime}(\mathrm{M})^{*} \\
6^{\prime \prime \prime}(\mathrm{B})^{*} \\
16 \mathrm{~mm}(\mathrm{R})^{*} \\
10 \mathrm{~mm}(\mathrm{~L})\end{array}$ & $\begin{array}{l}1-2^{\prime \prime \prime}(\mathrm{N}) \\
\mathrm{i}^{\prime \prime \prime}(\mathrm{S})\end{array}$ & $1.5^{\prime \prime \prime}(\mathrm{N})$ & $\begin{array}{l}3.5^{\prime \prime \prime}(\mathrm{N}) \\
15-20 \mathrm{~mm}(\mathrm{M}))^{*} \\
12 \mathrm{~mm}(\mathrm{~L})\end{array}$ & $\begin{array}{l}5.5^{\prime \prime \prime}(\mathrm{B})^{*} \\
2.5^{\prime \prime \prime}(\mathrm{N}) \\
10 \operatorname{mm}(\mathrm{L})\end{array}$ & $\begin{array}{l}3^{\prime \prime \prime}(\mathrm{N}) \\
6.5^{\prime \prime \prime}\left(\mathrm{B}^{*}\right. \\
11 \mathrm{~mm}(\mathrm{~L})\end{array}$ & $\begin{array}{l}2-3^{\prime \prime \prime}(\mathrm{N}) \\
7^{\prime \prime \prime}(\mathrm{B})^{*} \\
11-15 \mathrm{~mm}(\mathrm{~L}) \\
22 \mathrm{~mm}(\mathrm{R})\end{array}$ & $\begin{array}{l}2-3^{\prime \prime \prime}(\mathrm{N}) \\
6^{\prime \prime \prime}(\mathrm{B})^{*} \\
9 \mathrm{~mm}(\mathrm{~L})\end{array}$ & $\begin{array}{l}2 . .^{\prime \prime \prime}(\mathrm{N}) \\
6^{\prime \prime \prime}(\mathrm{B})^{*} \\
9 \mathrm{~mm}(\mathrm{~L}) \\
20-25(\mathrm{Fr})^{*}\end{array}$ \\
\hline IV & $\begin{array}{l}4^{\prime \prime \prime \prime}(\mathbf{N}) \\
4^{\prime \prime \prime \prime(S)} \\
8-9^{\prime \prime \prime}(\mathbf{B})^{*} \\
19 \mathrm{~mm}(\mathrm{~L}) \\
26 \mathrm{~mm}(\mathrm{R})\end{array}$ & $5-6^{\prime \prime \prime}(\mathrm{N})$ & $4^{\prime \prime \prime}(\mathbf{N})$ & $\begin{array}{l}8^{\prime \prime \prime}(\mathrm{N}) \\
13^{\prime \prime \prime}(\mathrm{B})^{*} \\
30-35 \mathrm{~mm}(\mathrm{M})^{*} \\
22 \mathrm{~mm}(\mathrm{~L})\end{array}$ & $\begin{array}{l}7-8^{\prime \prime \prime}(\mathrm{N}) \\
16 \mathrm{~mm}(\mathrm{R}) \\
19 \mathrm{~mm}(\mathrm{~L})\end{array}$ & $\begin{array}{l}8^{\prime \prime \prime}(\mathrm{N}) \\
30-35 \mathrm{~mm}(\mathrm{R}) \\
21 \mathrm{~mm}(\mathrm{~L})\end{array}$ & * $\begin{array}{l}4-5^{\prime \prime \prime}(\mathrm{N}) \\
14^{\prime \prime \prime}(\mathrm{B} * \\
23 \mathrm{~mm}(\mathrm{~L}) \\
28-30 \mathrm{~mm} \mathrm{R})\end{array}$ & $\begin{array}{l}4-5^{\prime \prime \prime}(\mathrm{N}) \\
11.5^{\prime \prime \prime}(\mathrm{B})^{*} \\
20 \mathrm{~mm}(\mathrm{~L})\end{array}$ & $\left.\begin{array}{l}\multicolumn{1}{c}{\cdot{ }^{\prime}} \\
11.5^{\prime \prime \prime}(B)^{*} \\
19 \mathrm{~mm}(\mathrm{~L}) \\
25-28 \mathrm{~mm} \\
30-35 \mathrm{~mm}\end{array}\right\}^{(\mathrm{Fr}}$ \\
\hline $\mathrm{V}$ & $\begin{array}{l}8-10^{\prime \prime \prime}(\mathrm{N}) \\
26 \mathrm{~mm}(\mathrm{~L})\end{array}$ & $7^{\prime \prime \prime}(\mathrm{N})$ & $6^{\prime \prime \prime}(\mathrm{N})$ & $\begin{array}{l}13-15^{\prime \prime \prime}(\mathrm{N}) \\
40-45 \mathrm{~mm}(\mathrm{M}) * \\
37 \mathrm{~mm}(\mathrm{~L})\end{array}$ & $\begin{array}{l}12^{\prime \prime \prime}(\mathrm{N}) \\
31 \mathrm{~mm}(\mathrm{~L})\end{array}$ & $\begin{array}{l}13^{\prime \prime \prime}(\mathrm{N}) \\
35 \mathrm{~mm}(\mathrm{~L})\end{array}$ & $\begin{array}{l}12^{\prime \prime \prime}(\mathrm{N}) \\
39 \mathrm{~mm}(\mathrm{~L}) \\
40 \mathrm{~mm}(\mathrm{Fr})\end{array}$ & $\begin{array}{l}12^{\prime \prime \prime}(\mathrm{N}) \\
35 \mathrm{~mm}(\mathrm{~L}) \\
35 \mathrm{~mm}(\mathrm{R})\end{array}$ & $\begin{array}{l}12^{\prime \prime \prime}(\mathrm{N}) \\
33 \mathrm{~mm}(\mathrm{~L})\end{array}$ \\
\hline$\nabla I$ & $\begin{array}{l}14^{\prime \prime \prime}(\mathrm{N}) \\
14^{\prime \prime \prime}(\mathrm{B})^{*} \\
33 \mathrm{~mm}(\mathrm{~L}) \\
33 \mathrm{~mm}(\mathrm{R}) \\
25 \mathrm{~mm}(\mathrm{~T})\end{array}$ & $\left\{\begin{array}{l}10^{\prime \prime \prime}(\mathrm{X}) \\
18 \mathrm{~mm} \\
25 \mathrm{~mm}\end{array}\right\}(\mathrm{T})$ & $\mid \begin{array}{l}6-7^{\prime \prime \prime}(\mathrm{N}) \\
16 \mathrm{~mm}(\mathrm{~T})\end{array}$ & $\begin{array}{l}16^{\prime \prime \prime}(\mathrm{N}) \\
20^{\prime \prime \prime}(\mathrm{B})^{*} \\
43 \mathrm{~mm}(\mathrm{~L}) \\
38 \mathrm{~mm}(\mathrm{~T}) \\
50 \mathrm{~mm}(\mathrm{~T})^{*}\end{array}$ & $\begin{array}{l}16^{\prime \prime \prime}(\mathrm{N}) \\
36 \mathrm{~mm}(\mathrm{~L}) \\
31.5 \mathrm{~mm}(\mathrm{~T}) \\
36.5 \mathrm{~mm}(\mathrm{~T})^{*}\end{array}$ & $\mid \begin{array}{l}17^{\prime \prime \prime}(\mathrm{N}) \\
41 \mathrm{~mm}(\mathrm{~L}) \\
35 \mathrm{~mm}(\mathrm{~T}) \\
40 \mathrm{~mm}(\mathrm{~T})^{*}\end{array}$ & $\mid$\begin{tabular}{l|}
$17^{\prime \prime \prime}(\mathrm{N})$ \\
$21^{\prime \prime \prime}(\mathrm{B})$ \\
$48 \mathrm{~mm}(\mathrm{~L})$ \\
$49-50 \mathrm{~mm}(\mathrm{R})$ \\
$40 \mathrm{~mm}(\mathrm{~T})$ \\
$56 \mathrm{~mm}(\mathrm{~T}) *$
\end{tabular} & $\begin{array}{l}17^{\prime \prime \prime}(\mathrm{N}) \\
42 \mathrm{~mm}(\mathrm{~L}) \\
36 \mathrm{~mm}(\mathrm{~T}) \\
43 \mathrm{~mm}(\mathrm{~T}){ }^{*}\end{array}$ & $\begin{array}{l}16-17^{\prime \prime \prime}(\mathrm{N}) \\
37 \mathrm{~mm}(\mathrm{~L}) \\
33 \mathrm{~mm}(\mathrm{~T}) \\
43 \mathrm{~mm}(\mathrm{~T})^{*}\end{array}$ \\
\hline VII & $\mid \begin{array}{l}16^{\prime \prime \prime}(\mathrm{N}) \\
40 \mathrm{~mm}(\mathrm{~L})\end{array}$ & $12^{\prime \prime \prime}(\mathrm{N})$ & $9^{\prime \prime \prime}(\mathrm{N})$ & $\begin{array}{l}20-22^{\prime \prime \prime}(\mathrm{N}) \\
53 \mathrm{~mm}(\mathrm{~L})\end{array}$ & $\begin{array}{l}17^{\prime \prime \prime}(\mathrm{N}) \\
44 \mathrm{~mm}(\mathrm{~L}) \\
33 \mathrm{~mm}(\mathrm{R})\end{array}$ & $\begin{array}{l}18^{\prime \prime \prime}(\mathrm{N}) \\
51 \mathrm{~mm}(\mathrm{~L})\end{array}$ & $\begin{array}{l}19-21^{\prime \prime \prime}(\mathrm{N}) \\
50-60 \min (\mathrm{R}) \\
60 \mathrm{~mm}(\mathrm{~L})\end{array}$ & $\begin{array}{l}19-21^{\prime \prime \prime}(\mathrm{N}) \\
53 \mathrm{~mm}(\mathrm{~L})\end{array}$ & $\begin{array}{l}19^{\prime \prime \prime}(\mathrm{N}) \\
52 \mathrm{~mm}(\mathrm{~L})\end{array}$ \\
\hline VIII & $\begin{array}{l}17-18^{\prime \prime \prime}(\mathrm{N}) \\
15^{\prime \prime \prime}(\mathrm{B})^{*} \\
42 \mathrm{~mm}(\mathrm{~L})\end{array}$ & $15^{\prime \prime \prime}(\mathrm{N})$ & $10^{\prime \prime \prime}(\mathrm{N})$ & $\begin{array}{l}23-24^{\prime \prime \prime}(\mathrm{N}) \\
22^{\prime \prime \prime}(\mathrm{B})^{*} \\
40 \mathrm{~mm}(\mathrm{M}) \\
56 \mathrm{~mm}(\mathrm{~L})\end{array}$ & $\begin{array}{l}18-19^{\prime \prime \prime}(\mathrm{N}) \\
46 \mathrm{~mm}(\mathrm{~L})\end{array}$ & $\begin{array}{l}20-22^{\prime \prime \prime}(\mathrm{N}) \\
54 \mathrm{~mm}(\mathrm{~L}) \\
45 \mathrm{~mm}(\mathrm{R})\end{array}$ & $\begin{array}{l}24^{\prime \prime \prime}(\mathrm{N}) \\
24^{\prime \prime \prime}(\mathrm{B})^{*} \\
64 \mathrm{~mm}(\mathrm{~L})\end{array}$ & $\begin{array}{l}24^{\prime \prime \prime}(\mathbf{N}) \\
24^{\prime \prime \prime}\left(\mathbf{B}^{*}\right. \\
56 \mathrm{~mm}(\mathrm{~L})\end{array}$ & $\begin{array}{l}21-23^{\prime \prime \prime}(\mathrm{N}) \\
25 \mathrm{~mm}(\mathrm{~L})\end{array}$ \\
\hline IX & $\begin{array}{l}45 \mathrm{~mm}(\mathrm{~L}) \\
40 \mathrm{~mm}(\mathrm{R})\end{array}$ & - & - & $61 \mathrm{~mm}\left(\mathrm{~L}_{\mathrm{L}}\right)$ & $51 \mathrm{~mm}(\mathrm{~L})$ & $59 \mathrm{~mm}(\mathrm{~L})$ & $\begin{array}{l}70 \mathrm{~mm}(\mathrm{~L}) \\
70-75 \mathrm{~mm}(\mathrm{R}) \\
85-90 \mathrm{~mm}(\mathrm{R}) *\end{array}$ & $53 \mathrm{~mm}\left(\mathrm{~L}_{1}\right)$ & $\mid \begin{array}{l}60 \mathrm{~mm}(\mathrm{~L}) \\
70-75 \mathrm{~mm}(\mathrm{Fr}) * \\
50-60 \mathrm{~mm}(\mathrm{Fr})\end{array}$ \\
\hline $\mathbf{X}$ & $\begin{array}{l}20^{\prime \prime \prime}(\mathbf{N}) \\
16^{\prime \prime \prime}(\mathbf{P})^{*} \\
50 \mathrm{~mm}(\mathrm{~L})\end{array}$ & $16^{\prime \prime \prime}(\mathrm{N})$ & $11^{\prime \prime \prime}(\mathrm{N})$ & \begin{tabular}{|l|}
$26-28^{\prime \prime \prime}(\mathrm{N})$ \\
$27^{\prime \prime \prime}(\mathrm{B})^{*}$ \\
$71 \mathrm{~mm}(\mathrm{~L})$ \\
$81 \mathrm{~mm}(\mathrm{Sk})^{*}$
\end{tabular} & $\begin{array}{l}20^{\prime \prime \prime}(\mathrm{N}) \\
22^{\prime \prime \prime}(\mathrm{B})^{*} \\
55 \mathrm{~mm}(\mathrm{~L}) \\
72 \mathrm{~mm}(\mathrm{Sk})^{*}\end{array}$ & $\begin{array}{l}24^{\prime \prime \prime}(\mathrm{N}) \\
26^{\prime \prime \prime}(\mathrm{B})^{*} \\
55-60 \mathrm{~mm}(\mathrm{R}) \\
65 \mathrm{~mm}(\mathrm{~L}) \\
76 \mathrm{~mm}(\mathrm{Sk})\end{array}$ & $\mid \begin{array}{l}27-28^{\prime \prime \prime}(\mathrm{N}) \\
32^{\prime \prime \prime}(\mathrm{B}) * \\
85 \mathrm{~mm}(\mathrm{~L}) \\
94 \mathrm{~mm}(\mathrm{Sk})^{*}\end{array}$ & $\begin{array}{l}27-28^{\prime \prime \prime}(\mathrm{N}) \\
27^{\prime \prime \prime}(\mathrm{B})^{*} \\
70 \mathrm{~mm}(\mathrm{~L}) \\
85 \mathrm{~mm}(\mathrm{Sk})^{*}\end{array}$ & $\begin{array}{l}27-28^{\prime \prime \prime}(\mathrm{N}) \\
27^{\prime \prime \prime}(\mathbf{B})^{*} \\
69 \mathrm{~mm}(\mathrm{~L}) \\
83 \mathrm{~mm}(\mathrm{Sk})^{*}\end{array}$ \\
\hline $\begin{array}{l}\text { New- } \\
\text { born }\end{array}$ & $\left.\begin{array}{l}1^{\prime \prime} 7^{\prime \prime \prime}(\mathrm{G}) \\
51 \mathrm{~mm}(\mathrm{~L}) \\
43.5 \mathrm{~mm} \\
46 . \mathrm{mm}\end{array}\right\}(\mathrm{T})$ & $\left.\begin{array}{l}1^{\prime \prime} 6^{\prime \prime \prime}(G) \\
33 \mathrm{~mm} \\
34 \mathrm{~mm} \\
41 \mathrm{~mm} \\
46 \mathrm{~mm}\end{array}\right\}(\mathrm{T})$ & $\left\{\begin{array}{l}1^{\prime \prime} 2^{\prime \prime \prime}(\mathrm{G}) \\
29 \mathrm{~mm} \\
26.5 \mathrm{~mm}\end{array}\right\}(\mathrm{T})$ & $\mid \begin{array}{l}3^{\prime \prime \prime}(\mathrm{G})^{*} \\
65-80 \mathrm{~mm}(\mathrm{M})^{*} \\
63 \mathrm{~mm}(\mathrm{U}) \\
76 \mathrm{~mm}(\mathrm{~L}) \\
65-66 \mathrm{~mm}(\mathrm{~T}) \\
80-83 \mathrm{~mm}(\mathrm{~T})^{*}\end{array}$ & \begin{tabular}{|l|}
$2^{\prime \prime} 8^{\prime \prime}(\mathrm{G})^{*}$ \\
$52 \mathrm{~mm}(\mathrm{R})$ \\
$43-48 \mathrm{~mm}(\mathrm{U})$ \\
$61 \mathrm{~mm}(\mathrm{~L})$ \\
$54-55 \mathrm{~mm}(\mathrm{~T})$ \\
$60-61 \mathrm{~mm}(\mathrm{~T})^{*}$
\end{tabular} & $\begin{array}{l}2^{\prime \prime} 10^{\prime \prime \prime}(\mathrm{G})^{*} \\
55 \mathrm{~mm}(\mathrm{U}) \\
70 \mathrm{~mm}(\mathrm{~L}) \\
61-62 \mathrm{~mm}(\mathrm{~T}) \\
70-71 \mathrm{~mm}(\mathrm{~T})^{*}\end{array}$ & $\begin{array}{l}3^{\prime \prime} 6^{\prime \prime \prime}(\mathrm{G})^{*} \\
94 \mathrm{~mm}(\mathrm{~L}) \\
73-75 \mathrm{~mm}(\mathrm{~T}) \\
90-99 \mathrm{~mm}(\mathrm{~T})^{*}\end{array}$ & $\begin{array}{l}3^{\prime \prime \prime} 2^{\prime \prime \prime}(\mathrm{G})^{\prime \prime} \\
83 \mathrm{~mm}(\mathrm{~L}) \\
63-67 \mathrm{~mm}(\mathrm{~T}) \\
73-80 \mathrm{~mm}(\mathrm{~T})\end{array}$ & $\begin{array}{l}3^{\prime \prime} 1^{\prime \prime \prime}(\mathrm{G})^{*} \\
79 \mathrm{~mm}(\mathrm{~L}) \\
60-63 \mathrm{~mm}(\mathrm{~T})\end{array}$ \\
\hline
\end{tabular}

TABLE III. Landois's table (in $\mathrm{mm}$.).

\begin{tabular}{|c|c|c|c|c|c|c|c|c|c|}
\hline \multicolumn{2}{|c|}{ Fetal Age } & No. & Clavicle & $\begin{array}{c}\text { Hu- } \\
\text { merus }\end{array}$ & Radius & Uln & Femur & 'Tibia & Fibula \\
\hline New- & born Child & $\begin{array}{l}1 \\
2\end{array}$ & $\begin{array}{r}51 \frac{13-}{32} \frac{2}{3} \\
49-504\end{array}$ & $\begin{array}{l}78 \\
74 \frac{1}{\delta}\end{array}$ & $\begin{array}{l}61 \\
59 \frac{2}{3}\end{array}$ & $\begin{array}{l}71 \\
68 \frac{1}{2}\end{array}$ & $\begin{array}{l}97 \\
91 \frac{1}{2}\end{array}$ & $\begin{array}{l}86 \\
80\end{array}$ & 81 \\
\hline & Month & 1 & $49 \frac{1}{2}-50 \frac{1}{3}$ & 71 & $55 \frac{1}{8}$ & $61 \frac{1}{2}$ & 85 & 70 & 69 \\
\hline IX & Month & $\begin{array}{l}1 \\
2 \\
3\end{array}$ & $\begin{array}{l}461-48 \\
442-45 \frac{1}{2} \\
42 \frac{1}{2}-43 \frac{1}{2}\end{array}$ & $\begin{array}{l}63 \\
62 \\
58 \frac{1}{2}\end{array}$ & $\begin{array}{l}52 \frac{1}{53} \\
47 \\
47\end{array}$ & $\begin{array}{l}60 \frac{1}{6} \\
60 \frac{1}{2} \\
55 \frac{2}{3}\end{array}$ & $\begin{array}{l}72 \frac{1}{2} \\
71 \frac{1}{3} \\
65 \frac{1}{2}\end{array}$ & $\begin{array}{l}66 \frac{1}{2} \\
69 \\
59\end{array}$ & $\begin{array}{l}64 \\
60 \\
57 \frac{1}{3}\end{array}$ \\
\hline VuII & Month & $\begin{array}{l}1 \\
2 \\
3\end{array}$ & $\begin{array}{c}40-41 \frac{1}{3} \\
422-44 \\
40-44\end{array}$ & $\begin{array}{l}56 \frac{1}{5} \\
56 \frac{3}{3} \\
54\end{array}$ & $\begin{array}{l}\frac{473}{46} \\
46\end{array}$ & $\begin{array}{l}55 \\
533 \\
53 \frac{3}{3}\end{array}$ & $\begin{array}{l}644 \\
644 \frac{1}{2} \\
644\end{array}$ & $\begin{array}{l}57 \frac{1}{3} \\
57 \frac{3}{3} \\
53 \frac{1}{2}\end{array}$ & $\begin{array}{l}56 \frac{1}{2} \\
54 \\
53\end{array}$ \\
\hline VII & Month & $\begin{array}{l}1 \\
2 \\
3 \\
4 \\
5\end{array}$ & $\begin{array}{c}40-43 \\
38-40 \\
39-43 \frac{1}{3-432} \\
39-42 \frac{2}{2} \\
34-36\end{array}$ & $\begin{array}{l}54 \\
54 \\
54 \\
53 \\
49 \\
49\end{array}$ & $\begin{array}{l}46 \\
44 \frac{1}{3} \\
43 \frac{1}{2} \\
43 \frac{1}{2} \\
42\end{array}$ & $\begin{array}{l}53 \\
52 \\
50 \frac{1}{2} \\
50 \frac{3}{2} \\
46 \frac{3}{3}\end{array}$ & $\begin{array}{l}65 \\
61 \frac{1}{2} \\
61 \\
59 \frac{1}{2} \\
54 \frac{3}{3}\end{array}$ & $\begin{array}{l}56 \frac{3}{5} \\
552 \\
524 \\
54 \\
49\end{array}$ & $\begin{array}{l}54 \\
54 \\
53 \\
50 \frac{1}{2} \\
48\end{array}$ \\
\hline VI & Month & $\begin{array}{l}1 \\
2 \\
3 \\
4\end{array}$ & $\begin{array}{r}36 \frac{1}{2}-38 \\
34 \frac{1}{2}-36 \frac{1}{2} \\
33 \frac{1}{3}-34 \frac{1}{d} \\
32-35 \frac{1}{3}\end{array}$ & $\begin{array}{l}49 \\
49 \\
47 \frac{1}{2} \\
43\end{array}$ & $\begin{array}{l}40 \frac{1}{2} \\
40 \\
38 \\
38\end{array}$ & $\begin{array}{l}47 \frac{1}{12} \\
46 \frac{1}{2} \\
44 \\
44\end{array}$ & $\begin{array}{l}56 \\
53 \frac{1}{2} \\
51 \frac{1}{3} \\
51\end{array}$ & $\begin{array}{l}48 \frac{1}{2} \\
48 \\
45 \frac{1}{3} \\
47\end{array}$ & $\begin{array}{l}46 \frac{1}{2} \\
47 \\
44 \\
45 \frac{1}{2}\end{array}$ \\
\hline XXII & Week & 1 & $30-31$ & 41 & $34 \frac{1}{2}$ & $39 \frac{1}{8}$ & 45 & 39 & 38 \\
\hline XXI & Week & $\begin{array}{l}1 \\
2 \\
3 \\
4\end{array}$ & $\begin{array}{c}30 \frac{1}{3}-32 \\
30-32 \frac{1}{3} \\
29-32 \frac{2}{3} \\
30 \frac{1}{2}\end{array}$ & $\begin{array}{l}40 \frac{1}{2} \\
40 \\
39 \frac{1}{2} \\
39 \frac{1}{2}\end{array}$ & $\begin{array}{l}34 \frac{1}{2} \\
32 \frac{1}{2} \\
34 \\
32\end{array}$ & $\begin{array}{l}40 \\
38 \\
39 \frac{1}{3} \\
37\end{array}$ & $\begin{array}{l}43 \frac{1}{2} \\
45 \frac{1}{2} \\
45 \frac{1}{2} \\
43\end{array}$ & $\begin{array}{l}392 \\
38 \\
38 \frac{2}{8} \\
38\end{array}$ & $\begin{array}{l}38 \frac{1}{2} \\
36 \frac{1}{2} \\
38 \frac{2}{2} \\
36 \frac{1}{2}\end{array}$ \\
\hline $\mathrm{XX}$ & Week & 1 & $26-27$ & 37 & 31 & $35 \frac{1}{2}$ & $39 \frac{2}{3}$ & $35 \frac{1}{3}$ & $33 \frac{2}{3}$ \\
\hline XIX & Week & 1 & $24 \frac{1}{2}-26$ & 36 & $30 \frac{1}{2}$ & 35 & 39 & 34 & $32 \stackrel{2}{3}$ \\
\hline XVI & Week & 1 & $21 \frac{1}{2}-22$ & 27 & $22 \frac{1}{2}$ & $25 \frac{1}{2}$ & $27 \frac{1}{3}$ & 24 & $23 \frac{1}{2}$ \\
\hline $\mathbf{X V}$ & Week & 1 & $16 \frac{2}{3}$ & 23 & $18 \frac{2}{3}$ & $21=$ & 23 & 20 & $19 \frac{1}{3}$ \\
\hline XIV & Week & 1 & 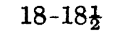 & $21 \frac{1}{3}$ & $17 \frac{1}{2}$ & $19 \frac{1}{3}$ & 21 & $18 \frac{2}{3}$ & $17 \frac{1}{3}$ \\
\hline XIII & Week & 1 & $18 \frac{1}{3}$ & 19 & $16 \frac{1}{2}$ & $18 \frac{1}{3}$ & $19 \frac{1}{2}$ & $16 \frac{2}{3}$ & 16 \\
\hline XII & Week & $\begin{array}{l}1 \\
2\end{array}$ & $\begin{array}{l}14 \frac{1}{3} \\
10 \frac{2}{3}\end{array}$ & $\begin{array}{l}17 \frac{1}{2} \\
15 \frac{1}{2}\end{array}$ & $\begin{array}{l}14 \frac{1}{8} \\
13\end{array}$ & $\begin{array}{l}15 \\
13 \frac{2}{3}\end{array}$ & $\begin{array}{l}17 \frac{1}{2} \\
15 \frac{1}{3}\end{array}$ & $\begin{array}{l}14 \frac{2}{9} \\
12 \frac{1}{2}\end{array}$ & $\begin{array}{l}13 \frac{1}{2} \\
11 \frac{2}{3}\end{array}$ \\
\hline XI & Week & $\begin{array}{l}1 \\
2\end{array}$ & 12 & $\begin{array}{l}14 \frac{1}{3} \\
18 \frac{1}{2}\end{array}$ & $\begin{array}{r}11 \frac{1}{6} \\
9 \frac{3}{4}\end{array}$ & $\begin{array}{l}11 \frac{1}{2} \\
10 \frac{1}{2}\end{array}$ & $\begin{array}{l}13 \\
13\end{array}$ & $\begin{array}{r}11 \\
8\end{array}$ & $\begin{array}{l}10 \\
9 \frac{1}{3}\end{array}$ \\
\hline $\mathrm{x}$ & Week & 1 & $8 \frac{3}{4}$ & $9 \frac{3}{4}$ & - & - & $9 \frac{1}{3}$ & 7 & $6 \frac{1}{2}$ \\
\hline IX & Week & 1 & $2 \frac{1}{2}$ & 2 & 2 & 2 & $1 \frac{1}{2}$ & $1 \ddagger$ & $1 \frac{1}{4}$ \\
\hline
\end{tabular}

TABLE II. Bones of the skull.

\begin{tabular}{|c|c|c|c|c|c|c|c|c|c|c|c|c|c|c|}
\hline \multirow{2}{*}{ Fetal Month } & \multicolumn{2}{|c|}{ Squamosum-occipital } & \multicolumn{2}{|c|}{ Basioccipital } & \multicolumn{2}{|c|}{ Parietal } & \multicolumn{2}{|c|}{ Frontal } & \multicolumn{2}{|c|}{ Squamosal } & \multicolumn{2}{|c|}{ Maxilla } & \multicolumn{2}{|c|}{ Zygomatic } \\
\hline & Height & Width & Length & w. & H. & w. & н. & W. & н. & w. & H. & w. & H. & w. \\
\hline II & - & - & - & - & - & - & $1.5^{\prime \prime \prime}(\mathrm{N})$ & $1^{\prime \prime \prime}(\mathrm{N})$ & - & - & $0.5^{\prime \prime \prime}(\mathrm{N})$ & $0.7^{\prime \prime \prime}(\mathrm{N})$ & - & - \\
\hline III & $2^{\prime \prime \prime}(\mathrm{N})$ & $3^{\prime \prime \prime}(\mathbf{N})$ & - & - & $6-9^{\prime \prime \prime}(\mathrm{N})$ & $3-8^{\prime \prime \prime}(\mathrm{N})$ & $\begin{array}{l}3^{\prime \prime \prime \prime}(\mathrm{N}) \\
1-1.5^{\prime \prime \prime}(\mathrm{N})\end{array}$ & $\begin{array}{l}2.5^{\prime \prime \prime}(\mathrm{N}) \\
2.5^{\prime \prime \prime}(\mathrm{S})\end{array}$ & $1^{\prime \prime \prime}(\mathrm{N})$ & $1.5^{\prime \prime \prime}(\mathrm{N})$ & $1.5^{\prime \prime \prime}(\mathrm{N})$ & $1.8-2^{\prime \prime \prime}(\mathrm{N})$ & - & - \\
\hline IV & $\begin{array}{l}1^{\prime \prime}(\mathrm{N}) \\
7 \mathrm{~mm}(\mathrm{~T})\end{array}$ & $\begin{array}{l}8-10^{\prime \prime \prime}(\mathrm{N}) \\
11 \mathrm{~mm}(\mathrm{~T})\end{array}$ & $1^{\prime \prime \prime}(\mathrm{N})$ & - & $\begin{array}{l}1^{\prime \prime} 2^{\prime \prime \prime}(\mathrm{N}) \\
15 \mathrm{~mm}(\mathrm{I})\end{array}$ & $\begin{array}{l}1^{\prime \prime} 2^{\prime \prime \prime}(N) \\
12 \mathrm{~mm}(\mathrm{~T})\end{array}$ & $\begin{array}{l}10-11^{\prime \prime \prime}(\mathrm{N}) \\
4^{\prime \prime \prime}(\mathrm{S}) \\
10 \mathrm{~mm}(\mathrm{~T})\end{array}$ & $\begin{array}{l}8-9^{\prime \prime \prime}(\mathrm{N}) \\
5^{\prime \prime \prime}(\mathrm{S}) \\
14 \mathrm{~mm}(\mathrm{~T})\end{array}$ & $\begin{array}{l}3-4^{\prime \prime \prime}(\mathrm{N}) \\
2.5 \mathrm{~mm}(\mathrm{~T})\end{array}$ & $\begin{array}{l}4-5^{\prime \prime \prime}(\mathrm{N}) \\
4 \mathrm{~mm}(\mathrm{~T})\end{array}$ & $\begin{array}{l}3-3.3^{\prime \prime \prime}(\mathrm{N}) \\
5 \mathrm{~mm}(\mathrm{~T})\end{array}$ & $\begin{array}{l}5-6^{\prime \prime \prime}(\mathrm{N}) \\
8.5 \mathrm{~mm}\end{array}$ & - & - \\
\hline $\mathrm{V}$ & $\begin{array}{l}1^{\prime \prime}(\mathrm{N}) \\
14 \mathrm{~mm}(\mathrm{~T})\end{array}$ & $\begin{array}{l}1^{\prime \prime}(\mathrm{N}) \\
21 \mathrm{~mm}(\mathrm{~T})\end{array}$ & $\mathbf{3}^{\prime \prime \prime}(\mathrm{N})$ & - & $\begin{array}{l}15-21^{\prime \prime \prime}(\mathrm{N}) \\
30 \mathrm{~mm}(\mathrm{~T})\end{array}$ & $\begin{array}{l}1^{\prime \prime} 3^{\prime \prime \prime}(\mathrm{N}) \\
28 \mathrm{~mm}(\mathrm{~T})\end{array}$ & $\begin{array}{l}1^{\prime \prime} 1^{\prime \prime \prime}-1^{\prime \prime} 3^{\prime \prime \prime}(\mathrm{N}) \\
22 \mathrm{~mm}(\mathrm{~T})\end{array}$ & $\begin{array}{l}11-13^{\prime \prime \prime}(\mathrm{N}) \\
21 \mathrm{~mm}(\mathrm{~T})\end{array}$ & $\begin{array}{l}4-5^{\prime \prime \prime}(\mathrm{N}) \\
4 \operatorname{mm}(\mathrm{T})\end{array}$ & $\begin{array}{l}5-7^{\prime \prime \prime}(\mathrm{N}) \\
8 \mathrm{~mm}(\mathrm{~T})\end{array}$ & $\begin{array}{l}5-6^{\prime \prime \prime}(\mathrm{N}) \\
7 \mathrm{~mm}(\mathrm{~T})\end{array}$ & $\begin{array}{l}7-3^{\prime \prime \prime}(\mathrm{N}) \\
18 \mathrm{~mm}(\mathrm{~T})\end{array}$ & - & - \\
\hline $\mathrm{VI}$ & $\begin{array}{l}1^{\prime \prime} 6^{\prime \prime \prime}(\mathrm{N}) \\
32 \mathrm{~mm}(\mathrm{~T})\end{array}$ & $\begin{array}{l}1^{\prime \prime} 4^{\prime \prime \prime}(\mathrm{N}) \\
40 \mathrm{~mm}(\mathrm{~T})\end{array}$ & $3.5^{\prime \prime \prime}(\mathrm{N})$ & $2.5^{\prime \prime \prime}(\mathrm{N})$ & $\begin{array}{l}21^{\prime \prime \prime}-2^{\prime \prime}(\mathrm{N}) \\
50 \mathrm{~mm}(\mathrm{~T})\end{array}$ & $\begin{array}{l}2^{\prime \prime}(\mathrm{N}) \\
46 \mathrm{~mm}(\mathrm{~T})\end{array}$ & $\begin{array}{l}1^{\prime \prime} 6^{\prime \prime \prime}(\mathbf{N}) \\
36 \text { mm }(\mathbf{T})\end{array}$ & $34 \mathrm{~mm}(\mathrm{~T})$ & $11 \mathrm{~mm}(\mathrm{~T})$ & $17 \mathrm{~mm}(\mathrm{~T})$ & $\begin{array}{l}7-8^{\prime \prime \prime}(\mathrm{N}) \\
13 \mathrm{~mm}(\mathrm{~T})\end{array}$ & $\begin{array}{l}9-10^{\prime \prime \prime}(\mathrm{N}) \\
30 \mathrm{~mm}(\mathrm{~T})\end{array}$ & - & - \\
\hline VII & $\left.\begin{array}{l}1^{\prime \prime} 6^{\prime \prime \prime}(\mathrm{N}) \\
42 \mathrm{~mm} \\
43 \mathrm{~mm}\end{array}\right\}(\mathrm{T})$ & $1^{\prime \prime} 4^{\prime \prime \prime}(\mathrm{N})$ & - & - & - & - & $1^{\prime \prime} 6^{\prime \prime \prime}(\mathrm{N})$ & $1^{\prime \prime} 6^{\prime \prime \prime}(\mathrm{N})$ & $9-9.5^{\prime \prime \prime}(\mathrm{N})$ & - & $8^{\prime \prime \prime}(\mathrm{N})$ & $9-10^{\prime \prime \prime}(\mathrm{N})$ & - & - \\
\hline VIII & $\mathbf{2}^{\prime \prime}(\mathrm{N})$ & $\left.\begin{array}{l}2^{\prime \prime}(\mathrm{N}) \\
45 \mathrm{~mm} \\
49 \mathrm{~mm}\end{array}\right\}(\mathrm{T})$ & $5^{\prime \prime \prime}(\mathrm{N})$ & - & $69-65 \mathrm{~mm}(\mathrm{~T})$ & $61-64 \mathrm{~mm}(\mathrm{~T})$ & $\begin{array}{l}2^{\prime \prime}(\mathrm{N}) \\
48-51 \mathrm{~mm}(\mathrm{~T})\end{array}$ & $41-43 \mathrm{~mm}(\mathrm{~T})$ & $16-20 \mathrm{~mm}(\mathrm{~T})$ & $21-23 m m(T)$ & $18-19 \mathrm{~mm}(\mathrm{~T})$ & $36-41 \mathrm{~mm}(\mathrm{~T})$ & - & - \\
\hline IX & $54 \mathrm{~mm}(\mathrm{~T})$ & $61 \mathrm{~mm}(\mathrm{~T})$ & - & - & $71 \mathrm{~mm}(\mathrm{~T})$ & $67 \mathrm{~mm}(\mathrm{~T})$ & $54 \mathrm{~mm}(\mathbf{T})$ & $54 \mathrm{~mm}(\mathrm{~T})$ & $19 \mathrm{~mm}(\mathrm{~T})$ & $24 \mathrm{~mm}(\mathrm{~T})$ & $21 \mathrm{~mm}(\mathrm{~T})$ & $42 \mathrm{~mm}(\mathrm{~T})$ & - & - \\
\hline $\mathbf{X}$ & $\begin{array}{l}2^{\prime \prime} 4^{\prime \prime \prime}(\mathrm{N}) \\
63 \mathrm{~mm}(\mathrm{~T})\end{array}$ & $\begin{array}{l}2^{\prime \prime} 6^{\prime \prime \prime}(\mathrm{N}) \\
64 \mathrm{~mm}(\mathrm{~T})\end{array}$ & $6^{\prime \prime \prime}(\mathrm{N})$ & - & $\begin{array}{l}3^{\prime \prime} 3^{\prime \prime \prime}(\mathbf{N}) \\
84 \mathrm{~mm}(\mathrm{~T})\end{array}$ & $\begin{array}{l}3^{\prime \prime} 6^{\prime \prime \prime}(\mathrm{N}) \\
79 \mathrm{~nm}(\mathrm{~T})\end{array}$ & $\begin{array}{l}\mathscr{Z}^{\prime \prime}(\mathrm{N}) \\
57 \mathrm{~mm}(\mathrm{~T})\end{array}$ & $51 \mathrm{~mm}(\mathrm{~T})$ & $\begin{array}{l}11-13^{\prime \prime \prime}(\mathrm{N}) \\
22 \mathrm{~mm}(\mathrm{~T})\end{array}$ & $\begin{array}{l}1^{\prime \prime}(\mathrm{N}) \\
31 \mathrm{~mm}(\mathrm{~T})\end{array}$ & $\begin{array}{l}11-12^{\prime \prime \prime}(\mathrm{N}) \\
25 \mathrm{~mm}(\mathrm{~T})\end{array}$ & $\begin{array}{l}13-11^{\prime \prime \prime}(\mathrm{N}) \\
49 \mathrm{~mm}(\mathrm{~T})\end{array}$ & - & - \\
\hline New-born & $\left.\begin{array}{l}2^{\prime \prime}(\mathrm{G}) \\
64 \mathrm{~mm} \\
67 \mathrm{~mm} \\
70 \mathrm{~mm}\end{array}\right\}(\mathrm{T})$ & $\left.\begin{array}{l}1^{\prime \prime} 10^{\prime \prime \prime}(\mathrm{G}) \\
62 \mathrm{~mm} \\
66 \mathrm{~mm} \\
68 \mathrm{~mm}\end{array}\right\}(\mathrm{T})$ & - & - & $\left.\begin{array}{l}3^{\prime \prime} 3^{\prime \prime \prime}((x) \\
83 \mathrm{~mm} \\
84 \mathrm{~mm} \\
85 \mathrm{~mm}\end{array}\right\}(\mathrm{T})$ & $\left.\begin{array}{l}75 \mathrm{~mm} \\
79 \mathrm{~mm} \\
80 \mathrm{~mm}\end{array}\right\}(\mathrm{T})$ & $\left.\begin{array}{l}2^{\prime \prime} 3^{\prime \prime \prime}(\mathrm{G}) \\
60 \mathrm{~mm} \\
62 \mathrm{~mm} \\
66 \mathrm{~mm}\end{array}\right\}(\mathrm{T})$ & $\left.\begin{array}{l}1^{\prime \prime} 10^{\prime \prime \prime}(\mathrm{G}) \\
53 \mathrm{~mm} \\
56 \mathrm{~mm} \\
57 \mathrm{~mm}\end{array}\right\}(\mathrm{T})$ & $\left.\begin{array}{l}1^{\prime \prime}(\mathrm{G}) \\
20 \mathrm{~mm} \\
23 \mathrm{~mm} \\
24 \mathrm{~mm}\end{array}\right\}(\mathrm{T})$ & $\left.\begin{array}{l}29 \mathrm{~mm} \\
32 \mathrm{~mm} \\
32 \mathrm{~mm}\end{array}\right\}(\mathrm{T})$ & $\left.\begin{array}{l}1^{\prime \prime}(\mathrm{G}) \\
22 \mathrm{~mm} \\
24 \mathrm{~mm} \\
25 \mathrm{~mm}\end{array}\right\}(\mathrm{T})$ & $\left.\begin{array}{l}1^{\prime \prime} 1^{\prime \prime \prime}(G) \\
50 \mathrm{~mm} \\
5 \operatorname{lmm} \\
51 \mathrm{~mm}\end{array}\right\}(\mathrm{T})$ & $6^{\prime \prime \prime}(\mathbf{G})$ & $1^{\prime \prime}(G)$ \\
\hline
\end{tabular}


TABLE IV. Size of the fetal bones (in cm.).

\begin{tabular}{|c|c|c|c|c|c|c|c|c|c|c|c|c|c|c|c|c|c|c|c|c|c|c|c|c|c|c|c|c|c|c|c|c|c|c|c|c|c|c|c|c|c|c|c|c|c|}
\hline \multirow{4}{*}{ 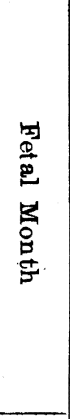 } & \multirow{4}{*}{ 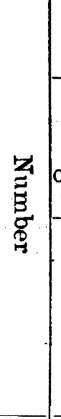 } & \multicolumn{26}{|c|}{ Bones of the skull } & \multicolumn{12}{|c|}{ Bones of the upper extremity } & \multicolumn{6}{|c|}{ Bones of the lower extr. } \\
\hline & & \multicolumn{2}{|c|}{$\begin{array}{c}\text { Squa- } \\
\text { mosum- } \\
\text { occipital }\end{array} \mid$} & \multicolumn{2}{|c|}{$\mid \begin{array}{c}\text { Basi- } \\
\text { occipital }\end{array}$} & \multicolumn{4}{|c|}{$\underset{\substack{\text { Squamosum- } \\
\text { frontal }}}{\text {. }}$} & \multicolumn{4}{|c|}{ Parietal } & \multicolumn{4}{|c|}{$\begin{array}{l}\text { Squamosum- } \\
\text { squamosal }\end{array}$} & \multicolumn{4}{|c|}{ Maxilla } & \multicolumn{4}{|c|}{ byomatic } & \multicolumn{2}{|c|}{$\begin{array}{c}\text { Body of } \\
\text { Sphe- } \\
\text { noid }\end{array}$} & \multirow{2}{*}{\multicolumn{2}{|c|}{$\begin{array}{c}\text { Clavicle } \\
\text { Length }\end{array}$}} & \multicolumn{4}{|c|}{ icapule } & \multirow{2}{*}{\multicolumn{2}{|c|}{$\mid$\begin{tabular}{c|}
$\begin{array}{c}\text { Hu- } \\
\text { merus }\end{array}$ \\
Length
\end{tabular}}} & \multirow{2}{*}{\multicolumn{2}{|c|}{\begin{tabular}{|l} 
Ulna \\
Length
\end{tabular}}} & \multirow{2}{*}{\multicolumn{2}{|c|}{\begin{tabular}{|l|} 
Radius \\
Length
\end{tabular}}} & $\mathrm{Fem}$ & & & & Fibr & \\
\hline & & 畄 & 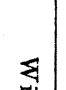 & 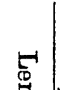 & 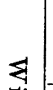 & Heig & & $\mathrm{Wi}$ & & $\begin{array}{l}\text { Sagi } \\
\text { diam }\end{array}$ & ittal & & & Heig & & Wid & & Heigl & & Wid & & Heig & & Wid & & $\vec{\Phi}$ & 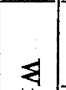 & & & Heig & & wid & & & & & & & & Leng & & Len: & & Leng & \\
\hline & & 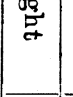 & 案 & $\stackrel{98}{5}$ & झ & 㿣 & 旨 & d. & 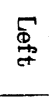 & $\mid$ & 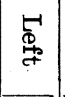 & 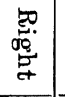 & 窵 & 先 & 点 & 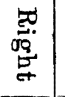 & $\underset{\$}{\stackrel{4}{*}}$ & 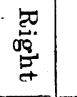 & 离 & 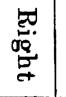 & 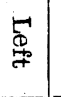 & 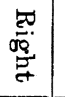 & 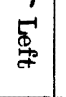 & 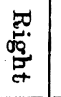 & $\underset{⿱ 乛 ⿰}{*}$ & 究 & 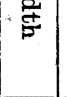 & 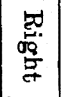 & $\underset{+}{\stackrel{5}{9}}$ & 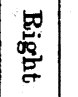 & 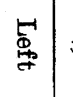 & بِّ & 点 & 量 & 点 & 誌 & 噧 & 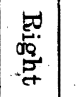 & 点 & 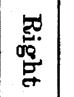 & 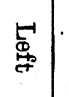 & . & 点 & 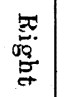 & $\underset{⿱ \mathbb{d}}{\stackrel{5}{*}}$ \\
\hline IV & i & 1.5 & 1.9 & 0.65 & & & & & & & 2.3 & & 1.6 & & 0.8 & & 1.0 & 0.9 & 0.8 & 1.0 & 1.0 & - & 0.7 & & 1.0 & - & - & 1.6 & 1.6 & 1.2 & & 0.95 & 1.0 & 2.2 & - & 2.0 & 1.9 & - & & & 2.1 & & & 1.8 & \\
\hline V & & & 4.4 & & 0.9 & & 4.8 & 4.8 & 4.8 & & \begin{tabular}{|l|}
5.8 \\
-
\end{tabular} & & 6.2 & 1.85 & 1.8 & & 2.8 & 1.8 & 1,9 & & 1.9 & 1.7 & 1.7 & 2.3 & 2.2 & 0.7 & 1.5 & 3.3 & 3.2 & 2.5 & 2.4 & 2.2 & & 4.6 & & 4.4 & 4.4 & 3.85 & 3.85 & 5.1 & 5.1 & 4.5 & 4.6 & 4.4 & \\
\hline VII & $\begin{array}{l}2 \\
3 \\
4\end{array}$ & $\begin{array}{l}4.4 \\
4.5 \\
4.8 \\
5.5\end{array}$ & $\begin{array}{l}5.0 \\
4.9 \\
5.4 \\
5.5\end{array}$ & $\begin{array}{l}1.1 \\
0.9 \\
1.0 \\
1.1\end{array}$ & $\begin{array}{l}1.1 \\
1.0 \\
1.15 \\
1.7\end{array}$ & $\begin{array}{c}4.0 .7 \\
4.8 \\
- \\
5.3\end{array}$ & $\begin{array}{l}5.8 \\
4.7 \\
-5.4 \\
5.4\end{array}$ & $\begin{array}{l}3.2 \\
3.9 \\
3.9\end{array}$ & $\begin{array}{l}3.1 \\
3.9 \\
4.0 \\
4.4\end{array}$ & $\begin{array}{r}6.0 \\
6.7 \\
6.2 \\
7.1\end{array}$ & $\mid \begin{array}{c}0.0 \\
6.2 \\
- \\
6.9\end{array}$ & $\begin{array}{c}6.0 \\
6.5 \\
6.3 \\
-7.8 \\
\end{array}$ & $\begin{array}{c}6.4 \\
6.4 \\
-2 \\
7.8\end{array}$ & $\begin{array}{l}\overline{1.8} \\
1.9 \\
18\end{array}$ & $\begin{array}{l}2.0 \\
1.9 \\
1.8\end{array}$ & $\begin{array}{l}\overline{2.6} \\
2.5 \\
2.7 \\
2.7\end{array}$ & $\begin{array}{l}2.5 \\
2.4 \\
2.9 \\
2.8 \\
2.8\end{array}$ & $\begin{array}{r}\overline{1.9} \\
2.0 \\
-7.05 \\
2.05\end{array}$ & $\begin{array}{l}7.9 \\
1.9 \\
2 \\
\mathbf{2 . 2}\end{array}$ & $\begin{array}{l}\overline{2.0} \\
1.9 \\
-2.1 \\
2.1\end{array}$ & $\begin{array}{l}2.0 \\
2.0 \\
2.0\end{array}$ & $\begin{array}{l}\overline{1.7} \\
1.6 \\
\overline{1.9}\end{array}$ & $\begin{array}{l}\overrightarrow{1.6} \\
1.6 \\
1.9\end{array}$ & $\begin{array}{l}2.1 \\
1.9 \\
-\end{array}$ & & $\mid \begin{array}{r}0.75 \\
0.75 \\
0.7\end{array}$ & $\begin{array}{c}-2.0 \\
1.3 \\
1.7 \\
1.5\end{array}$ & $\begin{array}{l}-3 . \\
3.1 \\
-\end{array}$ & $\begin{array}{l}3.3 \\
3.2 \\
-\end{array}$ & $\begin{array}{l}2.4 \\
2.7 \\
-\end{array}$ & $\begin{array}{l}2.5 \\
2.7 \\
-\end{array}$ & $\begin{array}{l}2.3 \\
2.0 \\
-\end{array}$ & & $\begin{array}{l}4.6 \\
4.4 \\
-\end{array}$ & $\begin{array}{l}4.6 \\
4.4 \\
-\end{array}$ & $\begin{array}{r}4.3 \\
4.2 \\
-\end{array}$ & $\begin{array}{l}4.3 \\
4.3 \\
-\end{array}$ & $\mid \begin{array}{l}3.9 \\
3.7 \\
-\end{array}$ & $\begin{array}{l}\overrightarrow{3.8} \\
3.7 \\
-\end{array}$ & $\begin{array}{r}5.1 \\
4.85 \\
-4 \\
\end{array}$ & $\begin{array}{r}5.1 \\
4.75 \\
-\end{array}$ & $\begin{array}{l}4.6 \\
4.3 \\
-\end{array}$ & $\begin{array}{l}4.5 \\
4.3 \\
-\end{array}$ & $\begin{array}{l}\overline{4.5} \\
4.2 \\
-\end{array}$ & 4.15 \\
\hline VIII & & $\begin{array}{l}4.9 \\
4.7\end{array}$ & & $\overline{1.1}$ & $\frac{1}{1.0}$ & $\begin{array}{l}4.5 \\
4.7\end{array}$ & $\begin{array}{l}4.3 \\
4.9 \\
4.9\end{array}$ & $\begin{array}{l}3.9 \\
4.1\end{array}$ & & & \begin{tabular}{|l|}
6.8 \\
6.0
\end{tabular} & $\begin{array}{l}7.0 \\
6.0\end{array}$ & & & 2.0 & $\begin{array}{l}3.0 \\
2.7\end{array}$ & $\begin{array}{l}3.0 \\
3.0\end{array}$ & \begin{tabular}{r|r|}
2.20 \\
2.2
\end{tabular} & $\frac{2.8}{2.0}$ & $\frac{2.1}{2.0}$ & $\frac{2.0}{2.1}$ & $\frac{1.0}{1.8}$ & $\frac{-9}{1.7}$ & 2.0 & $\frac{2.1}{2.0}$ & $\mid \frac{1.0}{1.0}$ & $\frac{1.0}{1.6}$ & 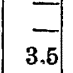 & -7. & $\overline{2.8}$ & 2.7 & $\exists$ & $\exists$ & 7 & $\overrightarrow{-1}$ & $\overline{-}$ & $\frac{7}{47}$ & $\begin{array}{l}4.2 \\
S\end{array}$ & & & 5.7 & 7.0 & & 二. & 7. \\
\hline & & 4.8 & 5.5 & & -1 & 4 & 4.6 & 4,0 & 4.2 & 6.8 & $\begin{array}{l}6.8 \\
\end{array}$ & 7.0 & 6.9 & & -1 & -1 & - & - & 1.7 & - & 2.1 & - & - & -1 & - & -1 & $\cdot-$ & 3.5 & -0 & $\cdot 2.8$ & 2.8 & 2.4 & 2.3 & 5.1 & 5.0 & 4.7 & 4.7 & 4.2 & 4.2 & & - & - & 4.9 & -1 & \\
\hline $\mathbf{x}$ & & & $\overline{7}$ & $\overline{1.0}$ & 1.4 & $\begin{array}{l}5.6 \\
5.5 \\
6.2\end{array}$ & $\begin{array}{l}5.5 \\
5.7 \\
6.1\end{array}$ & $\begin{array}{l}4.8 \\
4.9 \\
4.5\end{array}$ & $\begin{array}{l}4.8 \\
4.8 \\
4.6\end{array}$ & $\begin{array}{l}8.4 \\
8.4 \\
7.8\end{array}$ & \begin{tabular}{|l|}
7.8 \\
8.6 \\
7.5
\end{tabular} & $\begin{array}{l}7.9 \\
8.9 \\
8.4\end{array}$ & $\begin{array}{l}7.7 \\
9.0 \\
8.4\end{array}$ & 3.0 & 3.0 & 3.6 & $3 . \overline{-1}$ & $\begin{array}{l}2.7 \\
2.7\end{array}$ & $\begin{array}{l}2.5 \\
2.5 \\
.24\end{array}$ & $\mid \begin{array}{l}2.4 \\
2.6\end{array}$ & $\begin{array}{l}2.4 \\
2.4\end{array}$ & $\overline{2.0}$ & $\overrightarrow{2.0}$ & $\overrightarrow{2.6}$ & $\overrightarrow{2.8}$ & $=$ & 三 & 4.1.7. & & 3.7 & 3.7 & 3.0 & & $\begin{array}{r}5.95 \\
6.0\end{array}$ & $6 . \overline{-05}$ & $\begin{array}{l}\overrightarrow{5.7} \\
5.7\end{array}$ & $\begin{array}{l}7 \\
5.7 \\
5.7\end{array}$ & $\mid \begin{array}{l}7 \\
4.9 \\
5.0\end{array}$ & $\begin{array}{c}-7 \\
4.9 \\
5.05\end{array}$ & 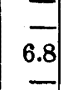 & 6.8 & $\overline{5.9}$ & & = & 5.8 \\
\hline & & - & - & - & - & - & - & - & - & 8.3 & - & 8.1 & - & - & - & - & - & - & -1 & - & - & - & - & - & - & - & - & - & -1 & - & - & - & - & - & - & - & - & - & - & -1 & - & - & - & -1 & \\
\hline & & 7 & 二 & 二 & 甘 & - & $\exists$ & & & 8.4 & \begin{tabular}{|c|}
8.2 \\
87
\end{tabular} & $\begin{array}{r}8.5 \\
94\end{array} \mid$ & $\begin{array}{r}8.5 \\
93\end{array}$ & - & ב & 二 & = & 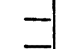 & = & 二 & 二 & 二 & - & - & - & - & - & - & - & - & - & - & - & - & - & - & - & - & - & - & - & - & - & - & \\
\hline & & 6.4 & 6.5 & $\exists$ & $\exists$ & $\exists$ & $\exists$ & & -7 & $\begin{array}{l}8.0 \\
8.0\end{array}$ & $\begin{array}{l}0.0 \\
7.9\end{array}$ & $\begin{array}{l}9.4 .4 \\
\end{array}$ & $\begin{array}{l}9.0 .5 \\
8.5\end{array}$ & $\exists$ & $\exists$ & $\exists$ & 二 & Z & $=$ & Z & $\exists$ & $\exists$ & 7 & $\exists$ & $\exists$ & $\exists$ & $\exists$ & $\exists$ & $\exists$ & $\exists$ & Z & $\exists$ & 二 & $\exists$ & 二 & Z & $\exists$ & $=$ & $\exists$ & 二 & $\exists$ & $\bar{z}$ & Z & 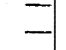 & \\
\hline & 6 & $\begin{array}{l}6.0 \\
6.3\end{array}$ & $\begin{array}{l}.0 .0 \\
6.0\end{array}$ & $\overrightarrow{1.3}$ & $\overrightarrow{1.6}$ & $\begin{array}{l}0.0 \\
6.4\end{array}$ & $\begin{array}{l}6.0 \\
6.5\end{array}$ & $\begin{array}{l}.8 .8 \\
5.3\end{array}$ & $\begin{array}{l}4.8 \\
5.2\end{array}$ & 8.1 & 8.0 & $\begin{array}{l}8.4 \\
8.5\end{array}$ & $\begin{array}{l}8.3 \\
8.2\end{array}$ & $\overrightarrow{2.7}$ & $2 . \overline{7}$ & $\overline{3.5}$ & $3 . \overline{3}$ & $\overrightarrow{2.5}$ & $\overrightarrow{2.6}$ & $\overrightarrow{2.6}$ & $\overrightarrow{2.6}$ & $\overrightarrow{2.0}$ & $\overrightarrow{2.0}$ & $\overrightarrow{2.7}$ & $\overrightarrow{2.5}$ & Z & Z & $\exists$ & $\exists$ & $\exists$ & 二 & 二 & Z & 二 & 二 & Z & Z & 二 & $\exists$ & 二 & 7 & 二 & 二 & $=$ & \\
\hline & 7 & - & & 1.4 & 1.5 & -1 & - & - & & -1 & - & - & - & - & 2.6 & - & 3.4 & - & - & - & - & - & 2.0 & - & 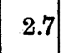 & $\begin{array}{c}1.2 \\
(\mathrm{~m})\end{array}$ & \begin{tabular}{l|l}
1.8 \\
\end{tabular} & - & - & - & - & - & - & -1 & - & - & - & - & - & - & -1 & - & - & - & \\
\hline & 8 & B. & $\begin{array}{l}6.3 \\
6.8\end{array}$ & $z$ & $\exists$ & - & ב & & & \begin{tabular}{|l|}
7.9 \\
7.5
\end{tabular} & \begin{tabular}{|l|}
7.5 \\
7.7
\end{tabular} & $\begin{array}{l}8.0 \\
7.9\end{array}$ & $\begin{array}{l}8.0 \\
8.0\end{array}$ & 二 & -1 & Z & = & $=$ & $=$ & = & - & 二 & 二 & - & Z & & -1 & Z & $\exists$ & Z & Z & $\exists$ & $=$ & 二 & 二 & $=$ & - & 二 & $\exists$ & $=$ & 二 & $I$ & $\exists$ & 二 & 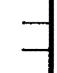 \\
\hline
\end{tabular}

TABLE V. Average of the cases of table IV (in cm.).

\begin{tabular}{|c|c|c|c|c|c|c|c|c|c|c|c|c|c|c|c|c|c|c|c|c|c|c|c|c|c|}
\hline \multirow{3}{*}{ Fetal Month } & \multicolumn{16}{|c|}{ Bones of the skull } & \multicolumn{6}{|c|}{ Bones of the upper extremity } & \multicolumn{3}{|c|}{ Bones of the lower extr. } \\
\hline & \multicolumn{2}{|c|}{$\begin{array}{c}\text { Squamosum- } \\
\text { occipital }\end{array}$} & \multicolumn{2}{|c|}{ Basi-occipital } & \multicolumn{2}{|c|}{ Frontal } & \multicolumn{2}{|c|}{ Parietal } & \multicolumn{2}{|c|}{ Squamosal } & \multicolumn{2}{|c|}{ Maxilla } & \multicolumn{2}{|c|}{ Zygomatic } & \multicolumn{2}{|c|}{$\begin{array}{c}\text { Body of } \\
\text { sphenoid }\end{array}$} & \multirow{2}{*}{$\begin{array}{c}\begin{array}{c}\text { Clar } \\
\text { vicle }\end{array} \\
\text { L. }\end{array}$} & \multicolumn{2}{|c|}{ Scapula } & \multirow{2}{*}{$\mid$\begin{tabular}{c|}
$\begin{array}{c}\text { Hu- } \\
\text { merus }\end{array}$ \\
L.
\end{tabular}} & \multirow{2}{*}{$\frac{0 \ln a}{\mathrm{~L} .}$} & \multirow{2}{*}{\begin{tabular}{|c|} 
Radius \\
IL \\
\end{tabular}} & \multirow{2}{*}{\begin{tabular}{|c|} 
Femur \\
L. \\
\end{tabular}} & \multirow{2}{*}{$\frac{\text { Tibia }}{\text { L. }}$} & \multirow{2}{*}{\begin{tabular}{|c|} 
Fibula \\
L.
\end{tabular}} \\
\hline & Height & Width & Length & Width & Height & Width & w. & н. & н. & w. & н. & w. & н. & W. & L. & w. & & н. & w. & & & & & & \\
\hline $\begin{array}{c}\text { IV } \\
\text { VI } \\
\text { VII } \\
\text { VIII } \\
\text { X } \\
\text { New-born }\end{array}$ & $\begin{array}{l}1.5 \\
5.6 \\
4.4 \\
5.0 \\
6.2 \\
6.6\end{array}$ & $\begin{array}{l}1.9 \\
4.4 \\
4.8 \\
5.0 \\
6.9 \\
6.4\end{array}$ & $\begin{array}{l}0.7 \\
1.0 \\
1.0 \\
1.1 \\
1.0 \\
1.4\end{array}$ & $\begin{array}{l}0.4 \\
0.9 \\
1.1 \\
1.4 \\
1.4 \\
1.6\end{array}$ & $\begin{array}{l}2.0 \\
4.8 \\
4.4 \\
4.8 \\
5.8 \\
6.1\end{array}$ & $\begin{array}{l}1.9 \\
4.8 \\
3.7 \\
4.1 \\
4.7 \\
5.0\end{array}$ & $\begin{array}{l}\mathbf{2 . 3} \\
5.8 \\
6.2 \\
6.7 \\
8.1 \\
8.0\end{array}$ & $\begin{array}{l}1.6 \\
6.2 \\
4.4 \\
6.9 \\
8.3 \\
8.4\end{array}$ & $\begin{array}{l}0.8 \\
1.8 \\
1.9 \\
2.0 \\
3.0 \\
2.7\end{array}$ & $\begin{array}{l}1.0 \\
2.7 \\
2.6 \\
2.9 \\
3.6 \\
3.5\end{array}$ & $\begin{array}{l}0.9 \\
1.9 \\
1.9 \\
2.0 \\
2.6 \\
2.6\end{array}$ & $\begin{array}{l}1.0 \\
1.9 \\
2.0 . \\
2.1 \\
2.5 \\
2.6\end{array}$ & $\begin{array}{l}0.7 \\
1.7 \\
1.6 \\
1.8 \\
2.0 \\
2.0\end{array}$ & $\begin{array}{l}1.0 \\
2.3 \\
2.0 \\
2.0 \\
2.7 \\
2.7\end{array}$ & $\begin{array}{l}\overline{0.7} \\
0.7 \\
1.0 \\
\overline{1.2} \\
(\mathrm{~m})\end{array}$ & $\begin{array}{l}\overline{1.5} \\
1.6^{\circ} \\
1.6 \\
1.8\end{array}$ & $\begin{array}{l}1.6 \\
3.3 \\
3.2 \\
3.5 \\
4.2 \\
-\end{array}$ & $\begin{array}{l}1.2 \\
3.5 \\
8.6 \\
2.8 \\
3.7 \\
\end{array}$ & $\begin{array}{l}1.0 \\
2.2 \\
2.2 \\
2.3 \\
3.0 \\
\end{array}$ & $\begin{array}{l}2.2 \\
4.6 \\
4.5 \\
5.0 \\
6.0\end{array}$ & $\begin{array}{l}2.0 \\
4.4 \\
4.3 \\
4.7 \\
5.7 \\
-\end{array}$ & $\begin{array}{l}3 . \overline{3} \\
3.8 \\
4.2 \\
5.0\end{array}$ & $\begin{array}{l}2.1 \\
5.1 \\
5.0 \\
5.7 \\
6.8 \\
\end{array}$ & $\begin{array}{l}1.9 \\
4.6 \\
4.4 \\
5.0 \\
6.0\end{array}$ & $\begin{array}{l}1.8 \\
4.4 \\
4.3 \\
4.8 \\
5.8 \\
-\end{array}$ \\
\hline
\end{tabular}

Giichiro Takata, On the Value of Bones as Diagnostic Material of Fetal Age. 
TABLE VI. Tutal average of the extremity-bones (in $\mathrm{mm}$.).

\begin{tabular}{|c|c|c|c|c|c|c|c|c|c|c|c|c|c|c|c|c|}
\hline \multirow{2}{*}{ 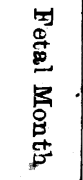 } & \multicolumn{2}{|c|}{ Clavicle } & \multicolumn{2}{|c|}{ Scapula } & \multicolumn{2}{|c|}{ Humerus } & \multicolumn{2}{|c|}{ Ulna } & \multicolumn{2}{|c|}{ Radius } & \multicolumn{2}{|c|}{ Femur } & \multicolumn{2}{|c|}{ Tibia } & \multicolumn{2}{|c|}{ Fibula } \\
\hline & C & 0 & $\stackrel{?}{C}$ & 0 & C & 0 & C & 0 & c & 0 & C & 0 & c & 0 & C & \\
\hline 11 & & & - & - & & 3 & 6 & - & & - & 17 & - & 16 & -1 & 9 & \\
\hline IU & 16 & 14 & & & $5-20$ & 11 & 17 & 10 & 15 & 9 & 21 & 10 & 16 & 8 & 20 & \\
\hline IV & 24 & 14 & 13 & 11 & 34 & & $0-35$ & 22 & - & 19 & 34 & 19 & 31 & 17 & 32 & \\
\hline$v$ & & 20 & 19 & & $\mid 0-45$ & 38 & - & 35 & - & 32 & - & 37 & - & 34 & - & \\
\hline $\mathrm{VI}$ & 38 & 32 & 28 & 19 & 52 & 43 & 40 & 40 & 37 & 39 & 57 & 40 & 43 & 43 & 43 & \\
\hline VII & - & 38 & 29 & 23 & -1 & 52 & 46 & 16 & - & 42 & - & 55 & - & 50 & -1 & \\
\hline IIII & 41 & 42 & 35 & 25 & $59 \mid$ & 52 & 49 & 19 & - & 48 & 65 & 61 & 65 & 57 & - & \\
\hline I & - & 43 & - & - & - & 61 & -1 & 19 & - & 515 & $5-90$ & 71 & - & & $0-75$ & \\
\hline $\mathrm{x}$ & 43 & 47 & 40 & 30 & 77 & 68 & 73 & 59 & 65 & 55 & 90 & 76 & 79 & 68 & 78 & \\
\hline & & & & 30 & & 68 & 76 & 62 & 68 & 53 & 96 & 84 & 81 & 74 & 80 & \\
\hline
\end{tabular}

TABLE VII. Total average of the skull bones (in $\mathrm{cm}$ ).

\begin{tabular}{|c|c|c|c|c|c|c|c|c|c|c|c|c|c|c|c|c|}
\hline \multirow{2}{*}{ 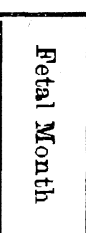 } & \multicolumn{2}{|c|}{$\begin{array}{c}\text { Squa- } \\
\text { mosusm- } \\
\text { occipital }\end{array}$} & \multicolumn{2}{|c|}{$\begin{array}{c}\text { Basi- } \\
\text { cccipital } \\
\end{array}$} & \multicolumn{2}{|c|}{ Parietal } & \multicolumn{2}{|c|}{ Frontal } & \multicolumn{2}{|c|}{$\begin{array}{l}\text { Squa- } \\
\text { mosal }\end{array}$} & \multicolumn{2}{|c|}{ Maxilla } & \multicolumn{2}{|c|}{$\begin{array}{c}\text { Zygoma- } \\
\text { tic } \\
\end{array}$} & \multicolumn{2}{|c|}{$\begin{array}{c}\text { Body } \\
\text { of of } \\
\text { sphenoid }\end{array}$} \\
\hline & $\mathrm{H}$ & $\mathrm{W}$ & L & w & 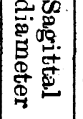 & 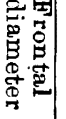 & H & $\mathrm{w}$ & $\mathrm{H}$ & $\mathrm{w}$ & $\mathrm{H}$ & w & H & W & $\mathrm{L}$ & $\mathrm{W}$ \\
\hline & & & & - & & - & & & - & - & & & - & - & & \\
\hline III & & & & & & & & 7 & & 5 & & $3-5$ & - & - & - & \\
\hline IV & & 18 & 5 & 4 & 23 & 20 & 17 & 18 & & 9 & 8 & 11 & 7 & 10 & - & - \\
\hline $\mathrm{V}$ & & ${ }^{24}$ & 8 & - & 37 & 32 & 27 & 27 & & 12 & 11 & 19 & - & - & - & - \\
\hline V I & 43 & 41 & 9 & ${ }^{8}$ & 54 & 54 & 42 & 41 & 15 & 22 & 18 & 21 & 17 & 23 & 7 & 1 \\
\hline VIII & 52 & 50 & 12 & 14 & 67 & 65 & 51 & 42 & 19 & 26 & 20 & 39 & 18 & 20 & 10 & 16 \\
\hline IX & 54 & 61 & - & - & 71 & 67 & 54 & 54 & 19 & 24 & 21 & 42 & - & - & - & - \\
\hline & 63 & 68 & 13 & 14 & 84 & 86 & 57 & 49 & 28 & 31 & 27 & 37 & 20 & 27 & - & \\
\hline & & 63 & 14 & & & 81 & 62 & 54 & 26 & 33 & 29 & 41 & 18 & 27 & 12 & \\
\hline
\end{tabular}

TABLE IX. Average of the cases of table VIII (in. gr.).

\begin{tabular}{|c|c|c|c|c|c|c|c|c|c|c|c|c|c|c|c|c|c|c|c|}
\hline \multirow{3}{*}{ 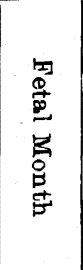 } & \multicolumn{11}{|c|}{ Bones of the skull } & \multicolumn{5}{|c|}{$\begin{array}{l}\text { Bones of the upper } \\
\text { extremity }\end{array}$} & \multicolumn{3}{|c|}{$\begin{array}{l}\text { Bones of the } \\
\text { lower extr. }\end{array}$} \\
\hline & & ipital & \multirow{2}{*}{ 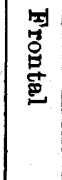 } & \multirow{2}{*}{ 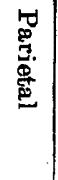 } & \multirow{2}{*}{\multicolumn{2}{|c|}{ 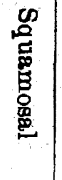 }} & \multirow{2}{*}{ 浐 } & \multirow{2}{*}{ 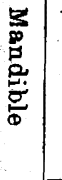 } & \multirow{2}{*}{ 管 } & \multicolumn{2}{|c|}{$\begin{array}{l}\text { Sphe- } \\
\text { noid }\end{array}$} & \multirow{2}{*}{ 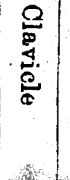 } & \multirow{2}{*}{ 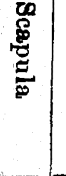 } & \multirow{2}{*}{\multicolumn{2}{|c|}{ 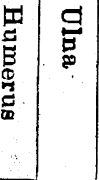 }} & \multirow{2}{*}{$\mid$} & \multirow{2}{*}{ 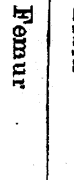 } & \multirow{2}{*}{ 宫 } & \multirow{2}{*}{ 琶 } \\
\hline & 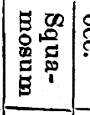 & : & & & & & & & & 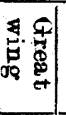 & 密 & & & & & & & & \\
\hline IV & & 2 & & & & & & & $z$ & - & - & & 5 & & & - & & 6 & \\
\hline $\mathbf{v I}$ & & 16 & 21 [ & 22 & & 0 & 28 & 38 & 17 & 28 & 13 & 15 & 33 & $65 \mid$ & 30 & & 127 & 84 & 19 \\
\hline VII & 93 & 20 & 26 & 12 & & 7 & 27 & 37 & 14 & 31 & 17 & 17 & 31 & 62 & 27 & 22 & 123 & 75 & 16 \\
\hline vIII & 137 & 30 & $32 \mid 12$ & \begin{tabular}{l|l}
33 & 19 \\
\end{tabular} & & 9 & 45 & 50 & 21 & 44 & 27 & 19 & 411 & 83 & 37 & 27 & 167 & 94 & 20 \\
\hline $\mathrm{x}$ & 376 & 61 & & 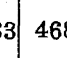 & & & & 112 & 39 & 95 & - & 60 & & 200 & 91 & 64 & 450 & 272 & 57 \\
\hline $\begin{array}{l}\text { Tew- } \\
\text { oorn }\end{array}$ & 409 & 51 & & 50 & *6 & & & 06 & 49 & & & - & - & - & - & - & - & - & - \\
\hline
\end{tabular}

TABLE VIII. Weight of the fetal bones (in gr.).

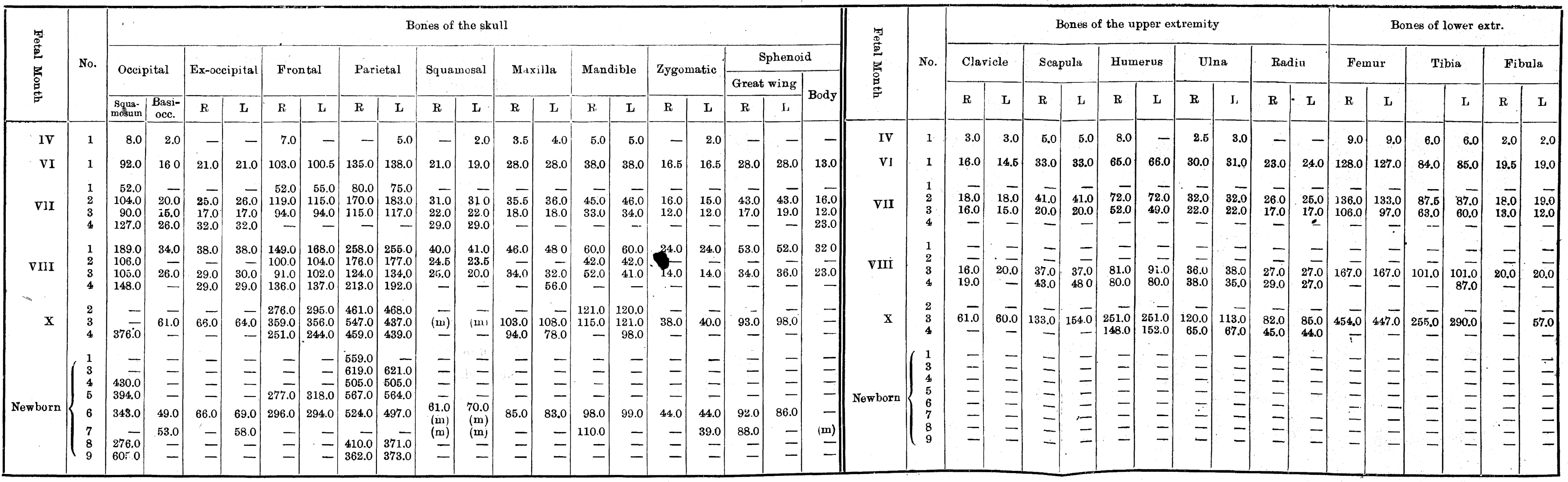

